\title{
Mapeamento da produção científica acerca do uso de biocompósitos nos processos de impressões 3D
}

Soluções em prol da sustentabilidade cercam as mais variadas pesquisas atualmente. Também é notório o impacto negativo que os resíduos sólidos causam ao meio ambiente, o que estimula o uso de matérias-primas originárias de fontes naturais, onde os biocompósitos aparecem como alternativa interessante. Novas tecnologias e modos de usá-las despertam a inventividade para estudos aprofundados, sendo a manufatura aditiva, também conhecida como impressão 3D, uma realidade de fácil acesso. Este estudo objetiva mapear as publicações científicas sobre o uso dos biocompósitos nos processos de impressão 3D disponíveis na base Scopus. Fez-se uso de técnicas bibliométricas para identificar a evolução dos temas no campo de pesquisa, os tópicos atuais e autores especialistas; criar um mapa do campo de estudo e descobrir as referências, periódicos e palavras-chave para pesquisas futuras. Para realizar este mapeamento utilizou-se do software SciMAT, ancorado em um método guiado por quatro fases: (i) deteç̧ão de temas de pesquisa; (ii) visualização de temas e ligações temáticas; (iii) descoberta de áreas temáticas; e (iv) análise de performance. Apenas 14 publicações foram encontradas sobre o assunto no período analisado. Identificou-se que o cluster 'TISSUEENGINEERING' é o único que se mantém ativo em todos os subperíodos analisados. Já o cluster 'FUSED-DEPOSITION-MODELING' é o mais representativo devido à quantidade de documentos associados ao tema. A partir dos resultados encontrados, observou-se que o uso de biocompósitos nos processos de impressões 3D é promissor e carece de pesquisa, sendo uma oportunidade para a revalorização dos resíduos sólidos através do uso da tecnologia aplicando e/ou desenvolvendo técnicas para a diminuição do impacto ambiental.

\section{Mapping of the scientific production on the usage of biocomposites in 3D printing processes}

\begin{abstract}
Solutions in support of sustainability have been present in the most diverse research fields. It is also notorious the negative impact that the solid residues cause to the environment, which stimulates the use of raw materials originated from natural sources, where the biocomposites appear as an interesting alternative. New technologies and means to use them arouse the ingenuity for more in-depth studies, being the additive manufacturing, also known as 3D printing, an easily accessible reality. This study aims at mapping the available scientific publications about the use of biocomposites in 3D printing processes in the database Scopus. Bibliometric techniques were used to identify the evolution of the themes in the research field, the current topics and the specialist authors; to create a map of the field of study and to find the references, journals and keywords for future researches. In order to carry out this scientific mapping, the software SciMAT was used, with basis on a method guided through four phases: (i) detection of research themes; (ii) visualization of themes and thematic networks; (iii) identification of the thematic areas; and (iv) performance analysis. Only 14 publications related to the topic were found in the analyzed period. It was identified that the cluster 'TISSUE-ENGINEERING' is the only one that maintains itself active in all the analyzed sub-periods. The cluster 'FUSED-DEPOSITION-MODELING', in turn, is the most representative due to the quantity of documents associated to the theme. Based on the results obtained, it was observed that the use of biocomposites in 3D printing processes is promising and under-researched, being an opportunity for the revaluation of the solid residues through the use of technology, applying or/and developing techniques for the reduction of the environmental impact.
\end{abstract}

Keywords: Biocomposites; Sustainable solutions; 3D printing; Additive Manufacturing.

Topic: Engenharia da Sustentabilidade e Meio Ambiente

Reviewed anonymously in the process of blind peer.
Received: $12 / 12 / 2019$

Approved: 13/01/2020
André Luiz Emmel Silva (iD

Universidade de Santa Cruz do Sul, Brasil

http://lattes.cnpq.br/1794234318779801

http://orcid.org/0000-0003-2499-1104

andresilva@unisc.br

Jorge André Ribas Moraes (ib

Universidade de Santa Cruz do Sul, Brasil

http://lattes.cnpq.br/9865102081473789

http://orcid.org/0000-0002-9505-8883

jorge@unisc.br

Lisianne Brittes Benitez (iD

Universidade de Santa Cruz do Sul, Brasil

http://lattes.cnpq.br/2698873972300996

http://orcid.org/0000-0002-0604-1407

lisianne@unisc.br

\section{Ezequiel Augusto Kaufmann (id \\ Universidade de Santa Cruz do Sul, Brasil \\ http://lattes.cnpq.br/9200705159170307 http://orcid.org/0000-0003-2230-3590 ezequielaugusto@mx2.unisc.br}

Leonardo Bertolin Furstenau (iD

Universidade de Santa Cruz do Sul, Brasil http://lattes.cnpq.br/4202946063932176 http://orcid.org/0000-0001-8984-7774 leonardofurstenau@mx2.unisc.br
Referencing this:

SILVA, A. L. E.; MORAES, J. A. R.; BENITEZ, L. B.; KAUFMANN, E. A.; FURSTENAU, L. B.. Mapeamento da produção científica acerca do uso de biocompósitos nos processos de impressões 3D. Revista Ibero Americana de Ciências Ambientais, v.11, n.1, p.236-250, 2020. DOI: http://doi.org/10.6008/CBPC2179-6858.2020.001.0022 


\section{INTRODUÇÃO}

Soluções sustentáveis devem ser cogitadas de forma pró-ativa, procurando meios e oportunidades que irão modificar a forma de pensar e agir da sociedade quanto à sustentabilidade, que deve ser entendida em seu sentido amplo: econômica, social, cultural e ambiental. O meio ambiente evoluiu do objeto de preservação para foco de ação sustentável. Novas práticas de gestão surgiram, como também emergiu uma nova 'economia ambiental', com a produção reciclada, a venda e o reaproveitamento de resíduos, gerando novos negócios, emprego e renda (MELO NETO et al., 2004). A manufatura verde pode ser entendida como o primeiro passo para o desenvolvimento da sustentabilidade, sendo projetada para ter um impacto positivo sobre o meio ambiente, reduzindo o consumo de energia e emissões de carbono (LE BOURHIS et al., 2013; FRAZIER, 2014). Na esfera científica, é notório o avanço nas pesquisas com materiais ambientalmente favoráveis. O que a pouco tempo era restrito ao uso da indústria automobilística e aeroespacial, agora ganha espaço nas mais diversas aplicações de engenharia.

O uso de polímeros e fibras sintéticas, produzidos a partir de fontes fósseis e com baixa velocidade de degradação no meio ambiente, tem sido reavaliado (RAMIRES, 2010). Crescem os estudos que visam o desenvolvimento de compósitos baseados em matéria-primas originárias de fontes naturais (MOHANTY et al., 2000; MENG et al., 2018), onde os biocompósitos aparecem como alternativa interessante. 0 termo biocompósito foi adotado para classificar os materiais que são compostos por, pelo menos, um componente de base biológica, por exemplo, bioplásticos, fibras naturais, nanocelulose (TARRÉS et al., 2018). São compósitos reforçados com fibras naturais, e se destacam pelas excelentes propriedades mecânicas, baixo custo, redução do impacto ambiental e efeito positivo na biodegradabilidade (MOHANTY et al., 2007; KARGARZADEH et al., 2017).

Resultados satisfatórios já foram encontrados em pesquisas com compósitos reforçados com casca de arroz (YAO et al., 2008; EL-KASSAS et al., 2013; KARGARZADEH et al., 2017), amido de milho com bambu e cânhamo (SHIBATA et al., 2008), fibras de sisal (CHOW et al., 2007; MOHANTY et al., 2007), bagaço de cana-de-açúcar (TRINDADE et al., 2004), palha de cevada, milho e trigo (MASLOWSKI et al., 2017), juta, banana e sisal (SINGH et al., 2017), fibras de curauá (SOUZA et al., 2015), e amido de mandioca (KARGARZADEH et al., 2017; VALENCIA et al., 2018). Estes biocompósitos são ecologicamente corretos, sendo denominados de 'eco-compósitos', adquirindo assim um grande potencial para serem os novos materiais do século XXI e consequentemente, uma solução parcial aos problemas ambientais globais (MILEO, 2011).

A impressão 3D também é encontrada na literatura como fabricação rápida, prototipagem rápida ou manufatura aditiva e, ao contrário dos processos de fabricação convencionais (subtrativos), cria a forma final por meio da adição de materiais (HUANG et al., 2013; RODRIGUES et al., 2017). Vem apresentando crescimento significativo nos últimos anos (LAPLUME et al., 2016; KUTZER et al., 2017; NAVARRETE et al., 2018), alavancada pela crescente disponibilidade e acessibilidade das tecnologias, expandindo sua abordagem para áreas anteriormente inexploradas (KITSON, 2014). Os resultados bem-sucedidos de pesquisas mostram um otimismo de que a manufatura aditiva tem um lugar significativo no futuro da 
fabricação (WONG et al., 2012), e um intenso interesse em ampliar o alcance das aplicações potenciais (LAURETO et al., 2017).

A realidade, cada vez mais próxima de fabricar produtos diretamente pelo consumidor (local print) com impressoras 3D é benéfica do ponto de vista do impacto ambiental, quando comparada com outras técnicas (WINNEBECK, 2011; KREIGER et al., 2013; WITTBRODT et al., 2015), tendo como vantagens a redução no consumo de material e energia (LE BOURHIS et al., 2013; YOON et al., 2014; FALUDI et al., 2015), redução na cadeia logística (FORD, 2014; FALUDI et al., 2015), redução da necessidade de ferramental (HOAG et al., 2012) e redução nas emissões de $\mathrm{CO}_{2}$ (BAUMERS et al., 2011; PETROVIC et al., 2011; GEBLER et al., 2014).

As aplicações das tecnologias de impressão 3D tornaram-se tão diversas quanto os tipos de materiais que podem ser utilizados para impressão (BADEN et al., 2015). Estudos já são conhecidos com metais, cerâmicas, polímeros, compósitos e sistemas biológicos (FRAZIER, 2014; FORD, 2014). É neste aspecto que muitos desafios ainda precisam ser superados, tanto no desenvolvimento de materiais quanto no seu uso para além das aplicações tradicionais (HOAG et al., 2012). Trata-se de um tema emergente e pouco estudado, o que indica a necessidade de reflexão sobre as potencialidades, restrições e possibilidades de difusão (RODRIGUES et al., 2017).

Com base no anteriormente exposto, o objetivo deste estudo foi realizar um mapeamento da produção científica acerca do uso de biocompósitos nos processos de impressões 3D. Para isso múltiplos objetivos foram traçados: (I) identificar a evolução dos temas no campo de pesquisa de biocompósitos utilizados em impressão 3D, os possíveis caminhos de pesquisa, os assuntos que receberam as maiores citações, os assuntos mais produtivos, bem como os temas com alto impacto científico; (II) descobrir tópicos atuais e autores especialistas no campo; (III) criar um mapa do campo de estudo; e (IV) descobrir as referências, periódicos e palavras-chave para pesquisas futuras.

\section{DISCUSSÃO TEÓRICA}

Esta pesquisa é de natureza aplicada, com objetivos exploratórios, implementada por meio de uma análise bibliométrica. Do ponto de vista da abordagem, é classificada como quali-quantitativa. Quantitativa, pois se buscou quantificar algumas variáveis referentes à produção científica sobre manufatura aditiva, impressão 3D e biocompósitos com a utilização de software de mapeamento científico. E qualitativa na medida em que teve caráter de exploração, de estimar, descobrir e compreender alguns tópicos relevantes ao tema.

Primeiramente, cinco critérios foram definidos: I - Critérios de escolha da base de dados: para esta pesquisa utilizou-se a base de dados Scopus por ser um dos bancos de dados bibliográficos mais confiáveis (VIEIRA et al., 2009; GOMEZ-JAUREGUI et al., 2014) e permitir a exportação de metadados para análise bibliométrica (COBO et al., 2011). II - Critérios de escolha do período: a pesquisa considera todas as publicações disponíveis na base Scopus até 2018. III - Critérios de escolha dos termos: em uma pesquisa bibliométrica, se um pesquisador pretende revelar os detalhes dos principais tópicos da pesquisa e suas relações, é necessário selecionar algumas palavras-chave como uma representação para os termos de busca 
(CHEN et al., 2016). Os termos de busca adotados foram: 'additive manufacturing' OR '3D printing' AND 'biocomposites'. IV - Critérios de escolha dos tipos de documentos: foi realizado o filtro para encontrar artigos que apresentassem algum dos termos de pesquisa no título, resumo e palavras-chave. $\mathbf{V}$ - Critérios de escolha do software de bibliometria: para o tratamento dos dados utilizou-se o software SciMAT (Science Mapping Analysis software Tool) desenvolvido por Cobo et al. (2012) pois permite a incorporação de métodos, algoritmos e medidas para todas as etapas do mapeamento científico, desde o pré-processamento até a visualização dos resultados. A estrutura utilizada para o mapeamento da produção científica desta pesquisa está apresentada na figura 1.

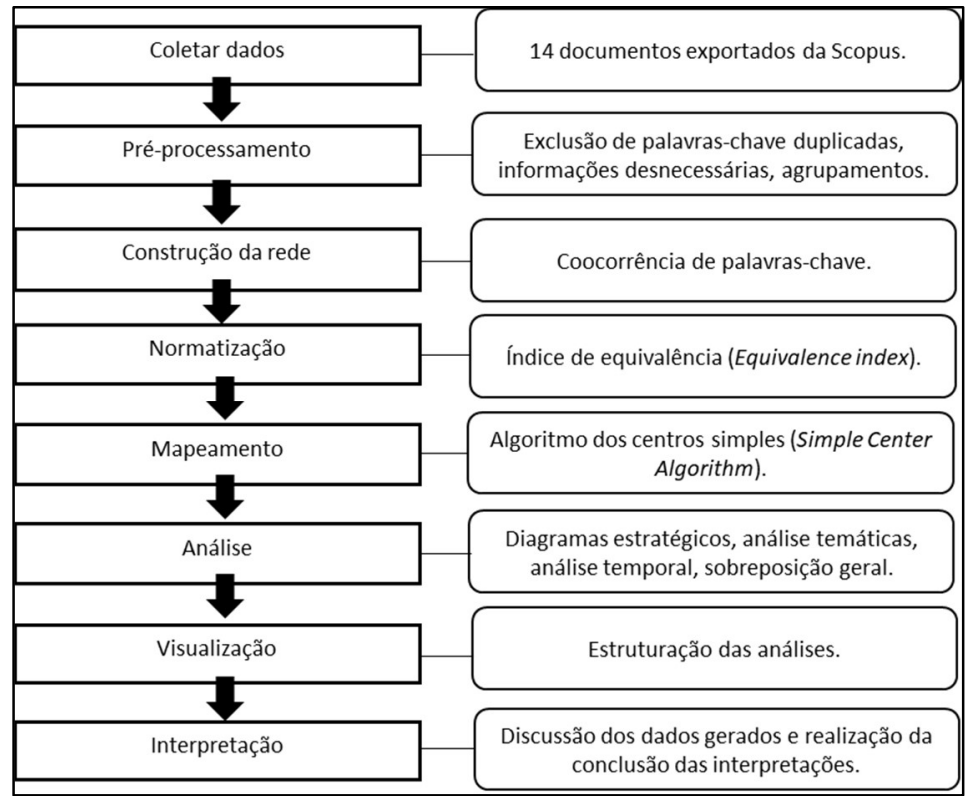

Figura 1: Estrutura para o mapeamento da produção científica. Fonte: adaptado de Cobo et al. (2012).

O método foi guiado por quatro fases distintas: (i) detecção de temas de pesquisa; (ii) visualização de temas e ligações temáticas; (iii) descoberta de áreas temáticas; e (iv) análise de performance.

Detecção de temas de pesquisa: nesta primeira fase foram utilizados todos os 14 documentos incluídos para análise bibliométrica. Os itens analisados foram palavras-chave e a extração das informações relevantes foi a frequência de coocorrência das palavras-chave, ou seja, quantidade de documentos em que as palavras aparecem juntas (СОВO et al., 2014). Para o cálculo de similaridade foi utilizado o índice de equivalência o qual calcula a força de ligação entre os cluster (COBO et al., 2014). O algoritmo clustering utilizado para detecção dos temas foi o algoritmo dos centros simples, o qual demonstra a força de ligação entre clusters (COBO et al., 2011).

Visualização de temas e ligações temáticas: Os temas obtidos através dos clusters formaram diagramas bidimensionais que possuem quatro quadrantes, baseados em valores de densidade (eixo y) e centralidade (eixo $\mathrm{x}$ ). A densidade mede a força interna de ligação, enquanto que a centralidade mede a intensidade da ligação de um cluster com outros clusters (CALLON et al., 1991). Neste contexto, os temas de pesquisa podem ser classificados em quatro grupos, mostrados no diagrama da Figura 2, também conhecido como diagrama estratégico ou diagrama de Callon (СOBO et al., 2012): 1) Temas motores, inclui tópicos bem desenvolvidos e importantes para o campo da pesquisa; 2) Temas básicos e transversais, contém tópicos 
importantes, mas fracamente estruturados 3) Temas emergentes ou declinando e 4) Altamente desenvolvidos e isolados.

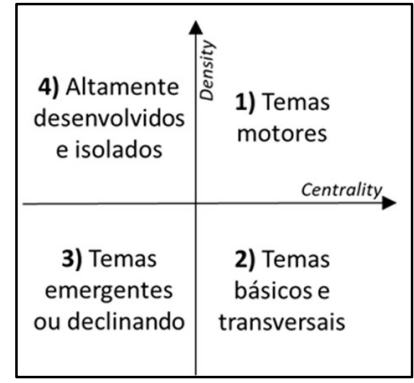

Figura 2: Diagramas estratégicos. Fonte: Cobo et al. (2012).

Descoberta de áreas temáticas: nesta terceira fase analisou-se a evolução dos temas de pesquisa a fim de evidenciar as principais áreas que evoluíram no campo de pesquisa em um determinado período de tempo, identificar origens, bem como as inter-relações. Para tanto, foi preciso construir um mapa de evolução com o índice de inclusão. A figura 3 apresenta um exemplo de um mapa da evolução temática. A linha sólida (linha 1 e 2 ) significa que os clusters conectados $\left(A^{1}\right.$ e $A^{2} ; B^{1}$ e $\left.B^{2}\right)$ compartilham o tema principal, enquanto que a linha tracejada (linha 3 ) caracteriza que os clusters $\left(B^{1}\right.$ e $\left.C^{1}\right)$ compartilham elementos que não são tema principal e quando não existe linha significa descontinuidade $\left(D^{1}\right.$ e $\left.D^{2}\right)$, sendo $D^{2}$ um novo cluster. A espessura das linhas é proporcional ao índice de inclusão, e o tamanho das esferas é proporcional ao número de documentos publicados associados com cada cluster (COBO et al., 2012).

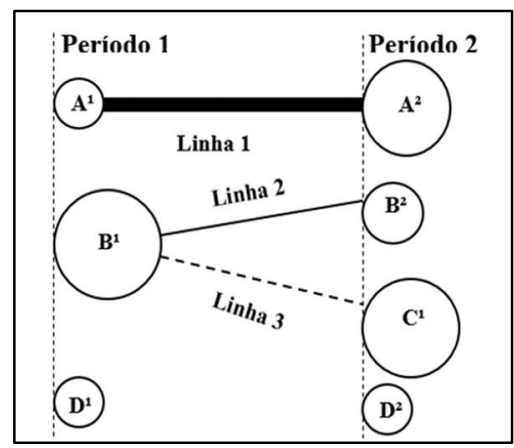

Figura 3: Exemplo de mapa de evolução temática. Fonte: Cobo et al. (2012).

Análise de performance: Nesta última fase, a contribuição de todo o campo de pesquisa foi medida (quantitativamente e qualitativamente), a fim de identificar a qualidade dos clusters e estabelecer as subáreas mais produtivas e de maior impacto.

\section{Pré-processamento dos dados para bibliometria}

Frequentemente dados exportados das bases de dados apresentam erros, logo é necessário realizar um pré-processamento a fim de garantir resultados bibliométricos de qualidade (COBO et al., 2012 ). Exportou-se todos os 14 documentos encontrados na análise bibliométrica da base de dados Scopus, os quais apresentaram um total de 185 palavras-chave. Em seguida um pré-processamento foi realizado excluindo-se duplicações de autores, referências, palavras-chave e documentos. Além disso, palavras como 'additive manufacturing', '3D printing' e 'biocomposites' foram excluídas, pois desejava-se identificar informações 
desconhecidas pelos autores. Da mesma forma, palavras com erros ortográficos foram corrigidas. Por fim, algumas palavras-chave foram agrupadas por representarem o mesmo conceito.

\section{MATERIAIS E MÉTODOS}

As 14 publicações encontradas datam do período de 2014 a 2018 (Figura 4). Assim, todo esse período foi considerado no desenvolvimento do mapeamento científico desta pesquisa. E para um entendimento da evolução temática, dividiu-se em três subperíodos: 2014 a 2016, 2017 e 2018. Foram analisados dois anos no primeiro subperíodo devido à baixa quantidade de publicações sobre o tema. A quantidade de documentos analisados em cada subperíodo são, respectivamente: 3, 4 e 7. Observou-se um aumento da quantidade de publicações ao longo dos anos.

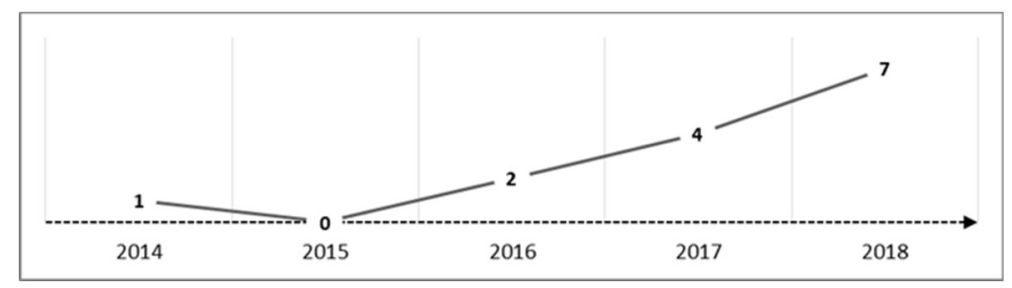

Figura 4: Quantidade de publicações na Scopus ao longo do tempo (2014 - 2018).

Foram 14 periódicos diferentes que publicaram sobre o tema, sendo um (01) artigo em cada: Composites Part B: Engineering, Journal of Supercritical Fluids, Carbohydrate Polymers, International Journal on Interactive Design and Manufacturing, Polymer Composites, Algal Research, Polymers, ACS Sustainable Chemistry and Engineering, ACS Omega, Composites Communications, Journal of Materials Chemistry B, Molecules, Materials and Design, e Applied Surface Science. Os autores que mais publicaram foram Johnny K. Melbø e Gary Chinga-Carrasco, ambos com três publicações cada. A figura 5 apresenta a lista completa das publicações por autor.

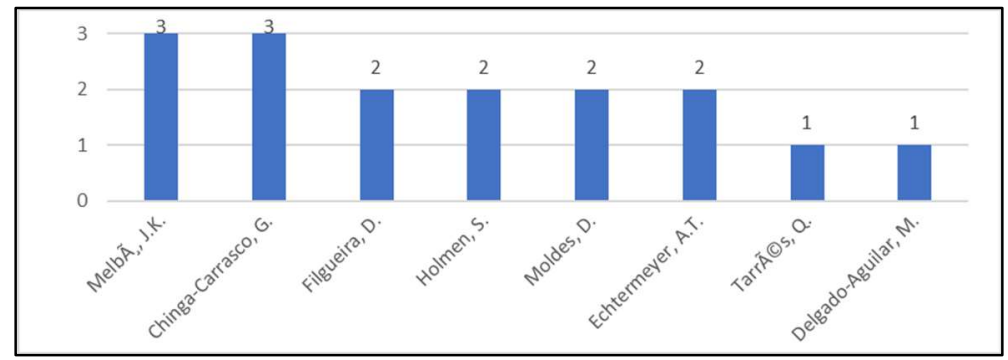

Figura 5: Autores que publicam estudos relacionados à biocompósitos para impressão 3D.

\section{RESULTADOS E DISCUSSÃO}

\section{Análise e discussões dos diagramas estratégicos}

A figura 6 mostra uma representação global dos resultados do algoritmo do centro simples. Os tópicos detectados em cada período são dispostos em um diagrama estratégico de acordo com sua centralidade e densidade. No primeiro subperíodo (figura 6a) pode-se observar quatro clusters. Neste subperíodo, 'TISSUE-ENGINEERING' se apresenta como um tema motor devido à sua alta centralidade (ligação com outros clusters) e densidade (tema altamente desenvolvido no campo de pesquisa). A 
engenharia de tecidos é o campo de estudo responsável pela reconstrução de novos órgãos e tecidos (HOLLISTER, 2005) com o suporte de polímeros bioreabsorvíveis (BARBANTI et al., 2005). O cluster 'EXTRUSION' apesar de sua densidade mediana, aparenta ser extremamente importante devido sua alta centralidade. Técnicas de extrusão foram utilizadas por autores como hot melt extrusion (HME) (XU et al., 2018); twin screw extrusion (NAVARRETE et al., 2018; MURPHY et al., 2018); extrusion freeforming (VAEZI, et al., 2016). Já o cluster 'WOOD' apresenta centralidade mediana e alta densidade. O cluster 'SELECTIVE-LASERSINTERING' pode ser tanto um tema emergente ou declinando devido à sua baixa centralidade e densidade.

No segundo subperíodo (figura 6b) 'TISSUE-ENGINEERING' deixa de ser um tema motor e passa a ser um tema altamente desenvolvido e isolado. Enquanto que 'WATER-CONTACT-ANGLE-MEASUREMENT' surge como o único tema motor. A técnica de medição do ângulo de contato com a água (WCA) é utilizada para medir a hidrofobicidade de fibras de polpas termomecânicas (TMP) (FILGUEIRA et al., 2017).

No terceiro subperíodo (figura 6c) 'TISSUE-ENGINEERING' volta a ser um tema motor. Por outro lado, surgem os clusters 'TWIN-SCREW-EXTRUSION' e 'THERMOMECHANICAL-PULPING-PROCESS' como principais temas motores. A Thermomechanical pulping é o processo de produção de polpa de madeira em um refinador de cavacos de madeira pré-cozidos (VENA, 2005). Além disso, apesar do cluster 'FUSEDDEPOSITION-MODELING' apresentar densidade mediana, este apresenta a maior centralidade de todos. O FDM (Modelagem por Deposição de Material Fundido) é um processo de extrusão de material que vem atraindo a atenção da comunidade científica nos últimos anos devido à sua alta capacidade de reinventar o processo de design (LE DUIGOU et al., 2016). Também conhecida como uma das técnicas mais populares de manufatura aditiva, o FDM é um método mais amplamente usado para fabricação de peças termoplásticas que são usadas principalmente como protótipos rápidos para testes funcionais, apresentando vantagens de baixo custo e pouco desperdício (NING et al., 2015).

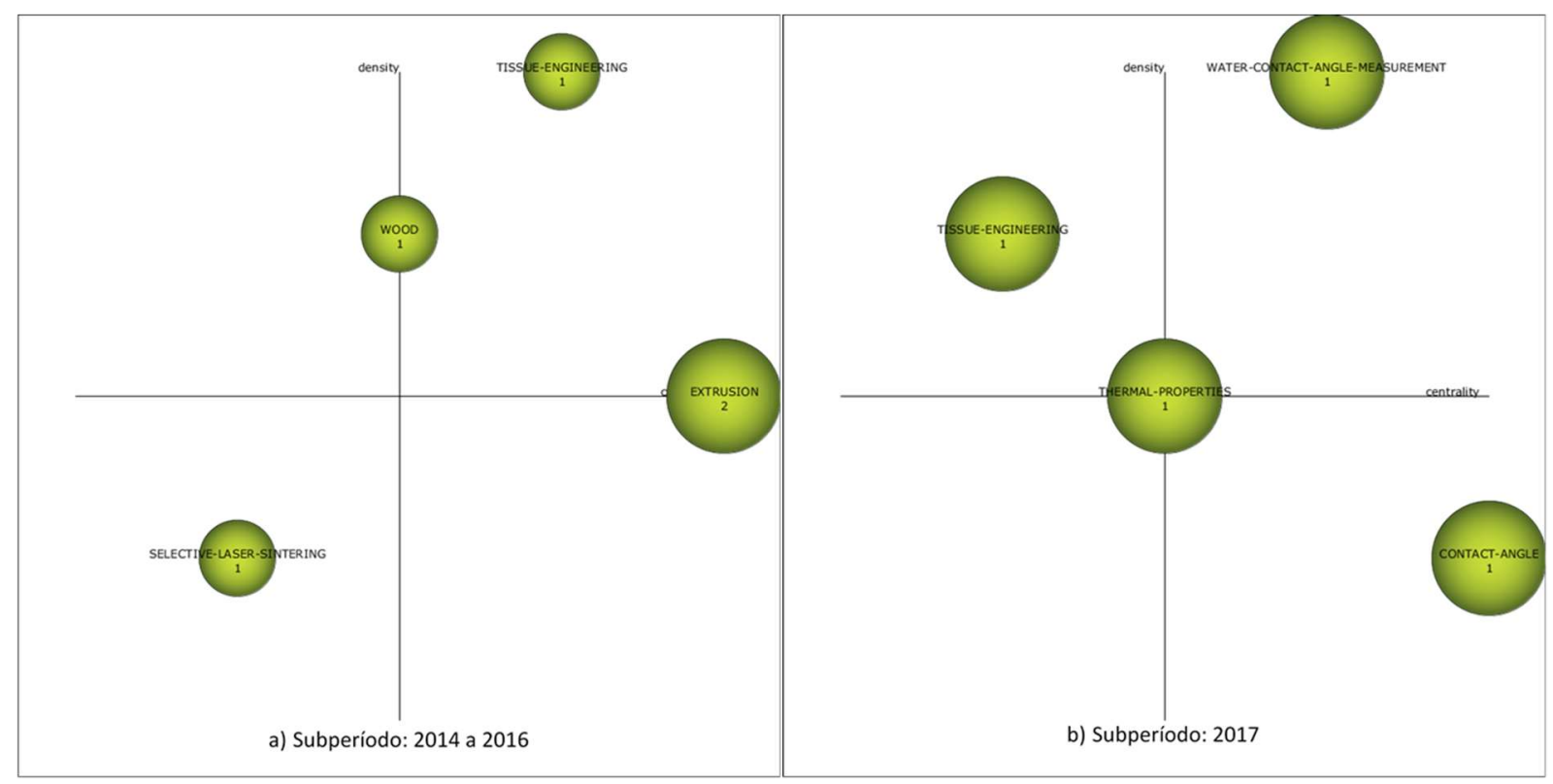




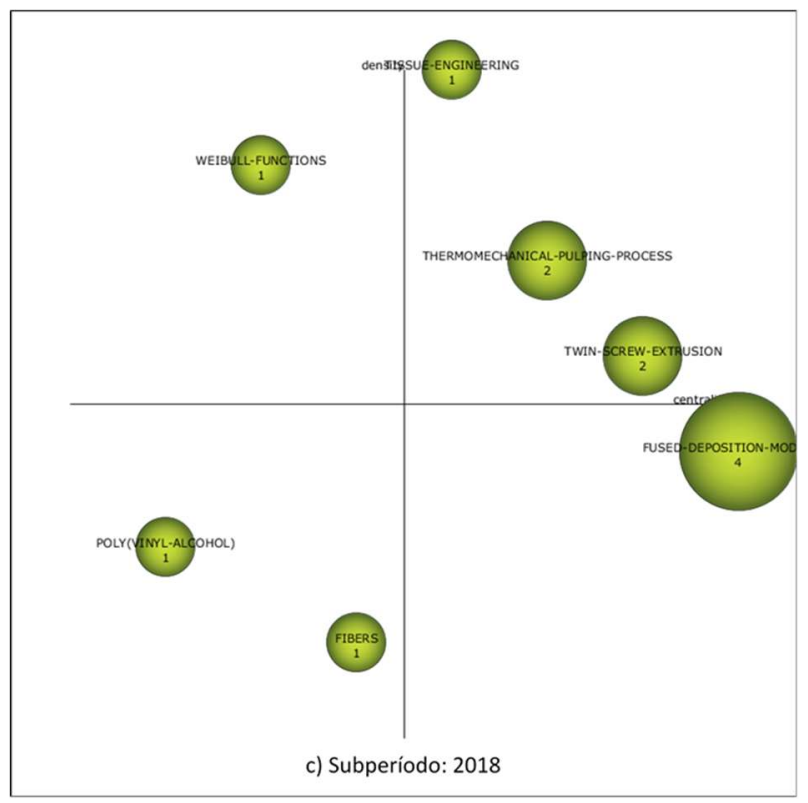

Figura 6: Diagramas estratégicos para o período de 2014 a 2018.

\section{Análise da evolução temática}

Ao analisar a evolução temática (figura 7) observa-se que o cluster 'TISSUE-ENGINEERING' é o único que se mantém nos três subperíodos. A engenharia de tecidos tem um grande impacto em aplicações médicas como regeneração de órgãos ou reparação de cartilagens (BEDIAN et al., 2017). A aplicação de biomateriais para projetar e fabricar suportes 3D (chamados de scaffolds) impulsionou o aparecimento de diversas pesquisas no campo de engenharia de tecidos (HUTMACHER, 2000). Sayyar et al. (2017) salientam que scaffolds com biocompatibilidade e biodegradabilidade apropriadas desempenham um papel fundamental no campo da engenharia de tecidos.

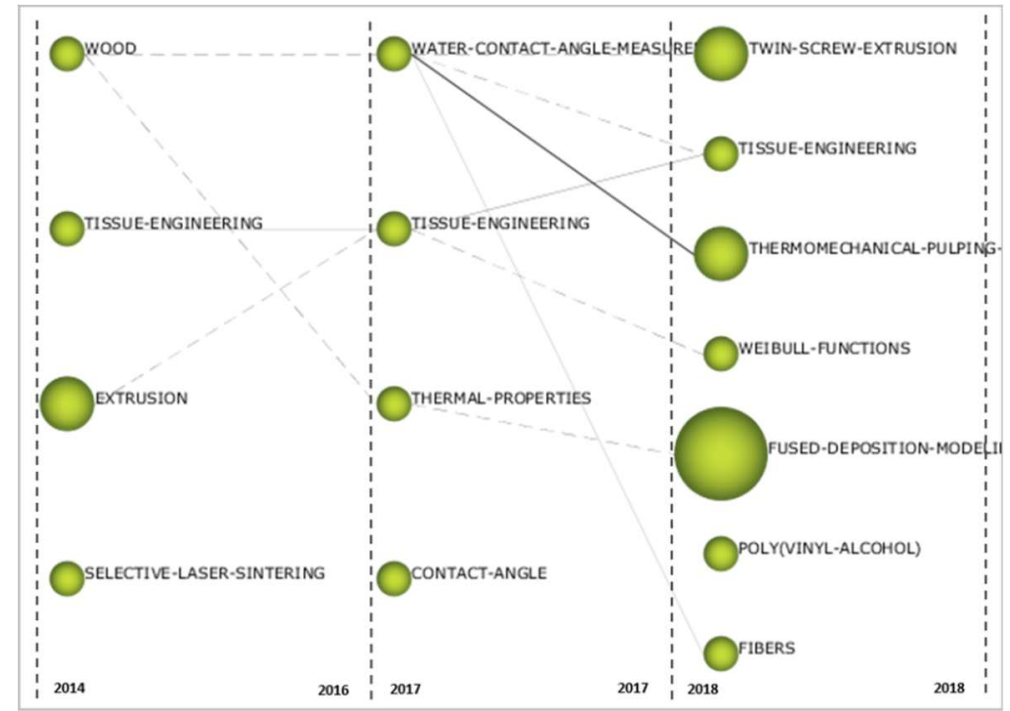

Figura 7: Evolução temática (2014 - 2018).

O cluster 'FUSED-DEPOSITION-MODELING' (figura 7) é o mais representativo devido à sua quantidade de documentos associados ao tema. O FDM é uma das tecnologias existentes para manufatura aditiva mais versátil e de fácil acesso e permite o desenvolvimento de scaffolds porosos que imitam a microestrutura do tecido vivo, os quais são utilizados na engenharia de tecidos (HUTMACHER, 2000). O equipamento é 
compacto e de baixo custo quando comparado à outras técnicas. Sua tecnologia de impressão é baseada em sistemas de impressoras de jato de tinta, que utilizam um cabeçote de impressão com centenas de orifícios que podem despejar milhares de gotículas por segundo (MEIRA et al., 2013). O processo consiste na adição de material camada por camada a partir de um arquivo pré-projetado (RAYNA et al., 2017; ZHANG et al., 2017). As informações são geradas em um arquivo CAD, e posteriormente convertidas em um arquivo STL (WONG et al., 2012), que contêm dados sobre a geometria do objeto. A impressão por FDM tem sido usualmente restrita a materiais termoplásticos comerciais, como o acrilonitrilo-butadieno-estireno (ABS) e o ácido poliláctico (PLA) (NAVARRETE et al., 2018).

O PLA é provavelmente o mais estudado dentre os polímeros biodegradáveis, sendo atualmente produzido a partir de matéria-prima renovável em escala industrial e competindo em custo com os commodities à base de petróleo (AVOLIO et al., 2018; XU et al., 2018). No entanto, o PLA possui baixa tenacidade o que o impede de ser adotado em aplicações duráveis. Esforços de pesquisa estão centrados na obtenção de produtos de PLA com propriedades melhoradas, através da mistura com outras resinas biodegradáveis (CASTRO-AGUIRRE et al., 2016; NAGARAJAN et al., 2016). O uso de fibras naturais é uma oportunidade real para satisfazer uma demanda crescente por materiais de alto desempenho projetados ecologicamente (RÉQUILÉ et al., 2018), e vem ganhando atenção crescente quando usadas como reforçador ou agente de enchimento em compósitos baseados em PLA (SCAFFARO et al., 2018). Matrizes termoplásticas, como PLA, quando combinadas com reforços biodegradáveis permite a produção de materiais totalmente biodegradáveis, os chamados biocompósitos (VILELA et al., 2013).

\section{Portfólio bibliográfico do assunto da pesquisa}

Dos 14 artigos mapeados nos processos anteriores, apenas um estava fora do escopo desta pesquisa: Vaezi et al. (2016), pois abordam técnicas de moldagem por extrusão e por compressão. Para todos os demais, uma síntese das contribuições identificadas em cada artigo é apresentada a seguir, organizada em ordem cronológica.

Liu (2014) realizou um estudo com o objetivo de fabricar um implante biocompósito de base óssea usando como matéria prima materiais biomédicos. Um biocomposto formado pela combinação de hidroxiapatite (HA), o sol de sílica, e tripolifosfato de sódio (STPP) foi gerado e usado para fabricar estruturas ósseas a partir da Impressão 3D por SLS (sinterização seletiva a laser). Os resultados indicam que os suportes fabricados pelo FDM com biopolímero como matéria-prima possuem maior porosidade e menor resistência à compressão. Além disso, os suportes fabricados pela 3DP e FDM têm menor resistência, enquanto os fabricados pela SLS têm maior resistência à compressão e menor porosidade. Uma vez que os suportes fabricados pela SLS têm maior resistência mecânica, a técnica SLS pode ser mais adequada para a fabricação de suportes ósseos na engenharia de tecidos.

Le Duigou et al. (2016) propuseram uma visão mais profunda dos efeitos dos parâmetros da impressão 3D por FDM na microestrutura de um biocompósito de madeira e suas consequências nas propriedades higromecânicas. A impressão de biocompósitos higromórficos por FDM permite uma mudança 
para a impressão 4D, uma vez que o material é capaz de evoluir ao longo do tempo em resposta a um estímulo externo. Os autores concluíram que a largura de impressão influencia as propriedades mecânicas dos biocompósitos de madeira, modificando sua microestrutura (porosidade). Aumentar a largura do filamento aumenta a porosidade, resultando em uma rápida e maior absorção de água, mas diminuindo a coesão do material. A impressão por FDM pode produzir materiais com propriedades mecânicas relativamente pobres e alta sensibilidade higroscópica.

Filgueira et al. (2017) realizaram um estudo que trata da modificação enzimática de fibras de polpas termomecânicas (TMP) para redução da absorção de água durante seu uso em filamentos de base biológica para impressão 3D. As fibras de TMP foram usadas como reforço para PLA, resultando em filamentos com menor poder de absorção de água, mas com boa adesão interfacial com a matriz polimérica, sendo então recomendado para impressão 3D por FDM.

Gupta et al. (2017) demonstraram o uso da hidroxiapatita como carga na matriz de PLA. Nanohidroxiapatita (n-HAP) é um fosfato de cálcio não tóxico bioativo, que constitui $60-70 \%$ dos ossos de mamíferos. Biocompósitos de PLA com n-HAP foram desenvolvidos com diferentes conteúdos de n-HAP. O material resultante foi processado como filamento, seguido de impressão 3D para produzir um osso do dedo para implante ortopédico. Os resultados revelam que a adesão e proliferação celular na superfície do biocompósito desenvolvido sustentam sua natureza biocompatível, possibilitando aplicações comerciais de engenharia biomédica e de alta temperatura.

Biswas et al. (2017) utilizaram nanopartículas híbridas de sílica e carbono (SCNPs) como reforço na proporção de 0,5, 1,0 e 1,5\% para melhorar as propriedades estruturais do polímero biodegradável Ecoflex. O Ecoflex é um biopolímero comercial disponível no mercado e fornecido pela BASF Corporation. O SCNPs foi extraído da casca de arroz pelo processo de pirólise simples. A impressora 3D do modelo Hyrel 30M foi usada para imprimir os filmes. Ensaios revelaram melhorias significativas nas propriedades térmicas e mecânicas do biopolímero Ecoflex devido à incorporação de 1,0\% em peso de SCNPs. Os resultados mostraram que a incorporação de nanopartículas interage com a matriz e aumenta a tenacidade e resistência do biopolímero.

Sayyar et al. (2017) realizaram uma revisão sobre trabalhos recentes relacionados a fabricação de estruturas usando biocompósitos baseados em grafeno, o qual possibilita construções com propriedades melhoradas para uma variedade de aplicações na área biomédica. Tarrés et al. (2018) usaram polietileno de base biológica (BioPE) misturadas com fibras de celulose na proporção de 10 e $20 \%$ para produzir biocompósitos. O BioPE é uma alternativa quimicamente idêntica ao polietileno da matéria-prima petroquímica. Em comparação com os BioPE puros, obteve-se um aumento da resistência à tração chegando a 127\% em algumas situações. Os biocompósitos formulados foram impressos em impressora 3D por FDM, e os resultados apontaram que a adição de fibras melhorou a qualidade de impressão e as propriedades mecânicas dos objetos impressos em 3D.

Ngo et al. (2018) utilizaram biocompostos de Polimetacrilato de metila (PMMA) enriquecidos com fosfato tricálcico ( $\beta$-TCP), preparados em impressão 3D por sinterização seletiva a laser (SLS), para serem 
usados como sistemas de liberação controlada de drogas (Controlled Drug Release Systems - CRDS). A CRDS envolve biomateriais internamente e externamente infundidos com vários produtos farmacêuticos antibióticos e anti-inflamatórios. Para formar o biocompósito, os pós cerâmicos foram pré-misturados com partículas de polímero selecionadas antes da impressão, mantendo assim a integridade estrutural do compósito, bem como a homogeneidade dos poros, o que é essencial para a distribuição uniforme do medicamento dentro do biomaterial. Os biocompósitos de PMMA/ $\beta$-TCP foram capazes de manter a estabilidade mecânica enquanto simultaneamente fornecem caráter biodegradável para mediar a liberação de moléculas de drogas bioativas. Os resultados comprovaram a viabilidade da impregnação de drogas dentro de estruturas biocompostas impressas em tecnologia 3D por SLS.

Xu et al. (2018) propuseram o uso de um biopolímero derivado de hemicelulose de madeira, para substituir parcialmente o PLA em Impressão 3D e investigar sua viabilidade. A hemicelulose é o segundo polissacarídeo mais abundante logo após a celulose, sendo tratadas como um fluxo secundário nas indústrias de processamento de biomassa. Os compósitos foram extrudados em filamentos seguidos por impressão 3D por FDM. Os resultados mostraram que quando o PLA foi substituído por até $20 \%$ hemicelulose de madeira, os compósitos mantiveram as propriedades mecânicas, sendo então uma alternativa de matéria-prima para impressão 3D por FDM.

Navarrete et al. (2018) demonstraram a obtenção de um compósito com uma matriz termoplástica reforçada com fibras naturais, neste caso a farinha de madeira. Os testes mostraram que um compósito com teor de fibra de $20 \%$ produziu um fio com as características adequadas para o processo de impressão por FDM. Os resultados dos ensaios indicam que as propriedades mecânicas do material compósito são de fato melhores do que o polímero virgem em amostras injetadas. Da mesma forma, o efeito do método de moldagem é significativo, e as peças obtidas em manufatura aditiva por FDM têm propriedades mecânicas muito menores em comparação com as peças obtidas pelos processos tradicionais de fabricação. No entanto, para algumas aplicações, essas características podem ser suficientes. Concluíram que é possível produzir filamentos customizados projetados para impressão 3D, os quais são até suscetíveis de serem utilizados na indústria de autopeças.

Murphy et al. (2018) produziram filamentos biocompósitos totalmente biodegradáveis para uso em impressão 3D. A celulose microcristalina foi usada como reforço para a matriz de PLA, na proporção de 1, 3 e 5\%. Para melhorar a compatibilidade com o PLA, a celulose foi modificada na superfície usando um agente de acoplamento de titanato. Os filamentos biocompostos foram preparados usando um processo de duas etapas: fundição de filme de PLA reforçado seguido por processo de extrusão. Os resultados apontaram que os filamentos de PLA reforçados com celulose foram impressos em 3D com sucesso usando uma técnica de FDM. Esses materiais oferecem a oportunidade de produção de protótipos biocompostos totalmente degradáveis para aplicações nos setores biomédico, automotivo e de construção.

Tran et al. (2018) sugeriram a criação de um biocompósito a partir da biomassa das microalgas usadas para extração de biodiesel, no entanto o mesmo não possui testes conclusivos em impressões 3D. Filgueira et al. (2018) misturaram polietileno de base biológica (BioPE) com fibras de celulose para fabricação de 
biocompósitos. Com o objetivo de reduzir a captação de água das fibras e dos correspondentes biocompósitos, o enxerto de compostos hidrofóbicos foi realizado por meio de um processo enzimático ecologicamente correto. Durante a impressão 3D por FDM, algumas amostras de filamentos de BioPE resultante apresentaram alta porosidade e variação de espessura, prejudicando a qualidade da peça. Os BioPE com baixo índice de fluidez melhoraram estas características. Já as amostras de BioPE misturadas com 10\% e 20\% de fibras de TMP mostraram suscetíveis a absorção de água. Essa absorção nos biocompósitos foi melhorada quando as fibras de celulose enzimaticamente hidrofilizadas foram utilizadas.

\section{CONCLUSÕES}

A partir do mapeamento científico identificou-se que as publicações sobre o uso de biocompósitos nos processos de impressões 3D são recentes e datam do período de 2014 a 2018. Apenas 14 publicações foram encontradas sobre o assunto, em 14 periódicos diferentes, com destaque para os autores Johnny K. Melb $\varnothing$ e Gary Chinga-Carrasco. Identificou-se que o cluster 'TISSUE-ENGINEERING' é o único que se mantém ativo em todos os subperíodos analisados e que o cluster 'FUSED-DEPOSITION-MODELING' é o mais representativo devido à quantidade de documentos associados ao tema.

Observou-se que o uso de biocompósitos nos processos de impressões 3D é promissor e carece de pesquisa, sendo uma oportunidade para a revalorização dos resíduos sólidos através do uso da tecnologia aplicando e/ou desenvolvendo técnicas para a diminuição do impacto ambiental. Assim, a utilização de materiais de origem não fósseis passa a ser uma alternativa atraente do ponto de vista econômico e ambiental, podendo ser usado como matéria prima das impressoras 3D que irão desenvolver, projetar ou prototipar novos produtos.

AGRADECIMENTOS: à Coordenação de Aperfeiçoamento de Pessoal de Nível Superior (CAPES) - Código de Financiamento 001.

\section{REFERÊNCIAS}

AVOLIO, R.; CASTALDO, R.; AVELLA, M.; COCCA, M. C.; GENTILE, G.; FIORI, S.; ERRICO, M. E.. PLA-based plasticized nanocomposites: Effect of polymer/plasticizer/filler interactions on the time evolution of properties. Composites Part B: Engineering, v.152, p.267-274, 2018. DOI: https://doi.org/10.1016/j.compositesb.2018.07.011

BADEN, T.; CHAGAS, A. M.; GAGE, G. J.; MARZULLO, T. C.; PRIETO-GODINO, L. L; EULER, T.. Open Labware: 3-D Printing Your Own Lab Equipment. PLOS Biology, v.13, n.5, 2015. DOI: https://doi.org/10.1371/journal.pbio.1002086

BARBANTI, S. H.; ZAVAGLIA, C. A. C.; DUEK, E. A. R.. Polímeros bioreabsorvíveis na engenharia de tecidos. Polímeros, São Carlos, v.15, n.1, p.13-21, 2005. DOI: http://dx.doi.org/10.1590/S0104-14282005000100006

BAUMERS, M.; TUCK, C.; BOURELL, D. L.; SREENIVASAN, R.; HAGUE, R.. Sustainability of additive manufacturing: measuring the energy consumption of the laser sintering process. Journal of Engineering Manufacture, v.225, n.12, p.2228-2239, 2011. DOI: https://doi.org/10.1177/0954405411406044

BEDIAN, L.; VILLALBA-RODRÍGUEZ, A. M.; HERNÁNDEZVARGAS, G.; PARRA-SALDIVAR, R.; IQBAL, H. M.. Bio-based materials with novel characteristics for tissue engineering applications-A review. International Journal of Biological Mmacromolecules, v.98, p.837-846, 2017. DOI: https://doi.org/10.1016/i.ijbiomac.2017.02.048

BISWAS, M. C.; JEELANI, S.; RANGARI, V.. Influence of biobased silica/carbon hybrid nanoparticles on thermal and mechanical properties of biodegradable polymer films. Composites Communications, v.4, p.43-53, 2017. DOI: https://doi.org/10.1016/i.coco.2017.04.005

CALLON, M.; COURTIAL, J. P.; LAVILLE, F.. Co-word analysis as a tool for describing the network of interactions between basic and technological research: The case of polymer 
chemsitry. Scientometrics, v.22, n.1, p.155-205, 1991.

CASTRO-AGUIRRE, F.; IÑIGUEZ-FRANCO, F.; SAMSUDIN, H.; FANG, X.; AURAS, R.. Poly (lactic acid) - Mass production, processing, industrial applications, and end of life. Advanced Drug Delivery Reviews, v.107, p.333-366, 2016. DOI: https://doi.org/10.1016/j.addr.2016.03.010

CHEN, G.; XIAO, L.. Selecting publication keywords for domain analysis in bibliometrics: A comparison of three methods. Journal of Informetrics, v.10, n.1, p.212-223, 2016. DOI: https://doi.org/10.1016/j.joi.2016.01.006

CHOW, C. P. L.; XING, X. S.; LI, R. K. Y.. Moisture absorption studies of sisal fibre reinforced polypropylene composites. Composites Science and Technology, v.67, n.2, p.306-313, 2007. DOI:

https://doi.org/10.1016/i.compscitech.2006.08.005

COBO, M. J.; LÓPEZ-HERRERA, A. G.; HERRERA-VIEDMA, E.; HERRERA, F.. An approach for detecting, quantifying, and visualizing the evolution of a research field: A practical application to the fuzzy sets theory field. Journal of Informetrics, v.5, n.1, p.146-166, 2011. DOI: https://doi.org/10.1016/j.joi.2010.10.002

COBO, M. J.; LÓPEZ-HERRERA, A. G.; HERRERA-VIEDMA, E.; HERRERA, F.. SciMAT: A new science mapping analysis software tool. Journal of the American Society for Information Science and Technology, v.63, n.8, p.16091630, 2012. DOI: https://doi.org/10.1002/asi.22688

COBO, M. J.; CHICLANA, F.; COLLOP, A.; ONA, J.; HERRERAVIEDMA, E.. A bibliometric analysis of the intelligent transportation systems research based on science mapping. IEEE Transactions on Intelligent Transportation Systems, v.15, n.2, p.901-908, 2014. DOI: https://doi.org/10.1109/TITS.2013.2284756

EL-KASSAS, A. M.; MOURAD, A. H. I.. Novel fibers preparation technique for manufacturing of rice straw based fiberboards and their characterization. Materials \& Design. v.50, p.757-765, 2013. DOI:

https://doi.org/10.1016/j.matdes.2013.03.057

FALUDI, J.; BAYLEY, C.; BHOGAL, S.; IRIBARNE, M.. Comparing environmental impacts of additive manufacturing vs traditional machining via life-cycle assessment. Rapid Prototyping Journal, v.21, n.1, p.14-33, 2015. DOI: https://doi.org/10.1108/RPJ-07-2013-0067

FILGUEIRA, D.; HOLMEN, S.; MELB $\varnothing$, J. K.; MOLDES, D.; ECHTERMEYER, A. T.; CHINGA-CARRASCO, G.. EnzymaticAssisted Modification of Thermomechanical Pulp Fibers to Improve the Interfacial Adhesion with Poly (lactic acid) for 3D Printing. ACS Sustainable Chemistry and Engineering, v.5, n.10, p.9338-9346, 2017. DOI: https://doi.org/10.1021/acssuschemeng.7b02351

FILGUEIRA, D.; HOLMEN, S.; MELB $\varnothing$, J. K.; MOLDES, D.; ECHTERMEYER, A. T.; CHINGA-CARRASCO, G.. 3D printable filaments made of biobased polyethylene biocomposites. Polymers, v.10, n.3, p.314, 2018. DOI: https://doi.org/10.3390/polym10030314

FORD, S. L. N.. Additive Manufacturing Technology: Potential Implications for U.S. Manufacturing Competitiveness.
Journal of International Commerce and Economics, 2014

FRAZIER, W. E.. Metal Additive Manufacturing: A Review. The International Journal of Advanced Manufacturing Technology, v.23, n.6, p.1917-1928, 2014. DOI: https://doi.org/10.1007/s11665-014-0958-z

GEBLER, M.; UITERKAMP, A. J. M. S.; VISSER, C.. A global sustainability perspective on 3D printing Technologies. Energy Policy, v.74, p.158-167, 2014. DOI: https://doi.org/10.1016/j.enpol.2014.08.033

GOMEZ-JAUREGUI, V.; GOMEZ-JAUREGUI, C.; MANCHADO, C.; OTERO, C.. Information management and improvement of citation indices. International Journal of Information Management, v.34, p.257-271, 2014. DOI: http://dx.doi.org/10.1016/j.ijinfomgt.2014.01.002

GUPTA, A.; PRASAD, A.; MULCHANDANI, N.; SHAH, M.; SANKAR, M. R.; KUMAR, S.; KATIYAR, V.. Multifunctional Nanohydroxyapatite-Promoted Toughened High-MolecularWeight Stereocomplex Poly (lactic acid)-Based Bionanocomposite for Both 3D-Printed Orthopedic Implants and High-Temperature Engineering Applications. ACS Omega, v.2, n.7, p.4039-4052, 2017. DOI: https://doi.org/10.1021/acsomega.7b00915

HOAG, C.; SPRADLING, D.; SHULMAN, H.. Introduction to Additive Manufacturing. Ceramic Industry Magazine, 2012.

HOLLISTER, S. J.. Porous scaffold design for tissue engineering. Nature materials, v.4, n.7, p.518, 2005. DOI: https://doi.org/10.1038/nmat1421

HUANG, P. H.; LIU, P.; MOKASDAR, A.; HOU, L.. Additive manufacturing and its societal impact: a literature review. The International Journal of Advanced Manufacturing Technology, v.67, n.5-8, p.1191-1203, 2013. DOI: https://doi.org/10.1007/s00170-012-4558-5

HUTMACHER, D. W.. Scaffolds in tissue engineering bone and cartilage. Biomaterials, v.21, n.24, p.2529-2543, 2000. DOI: https://doi.org/10.1016/S0142-9612(00)00121-6

KARGARZADEH, H.; JOHAR, N.; AHMAD, I.. Starch biocomposite film reinforced by multiscale rice husk fiber. Composites Science and Technology, v.151, p.147-155, 2017. DOI: https://doi.org/10.1016/i.compscitech.2017.08.018

KITSON, P. J.; MACDONELL, A.; TSUDA, S.; ZANG, H.; LONG, D. L.; CRONIN, L.. Bringing Crystal Structures to Reality by Three-Dimensional Printing. Crystal Growth Design, v.14, n.6, p.2720-2724, 2014. DOI: https://doi.org/10.1021/cg5003012

KREIGER, M.; PEARCE, J. M.. Environmental Life Cycle Analysis of Distributed Three-Dimensional Printing and Conventional Manufacturing of Polymer Products. ACS Sustainable Chem. Eng., v.1, n.12, p.1511-1519, 2013. DOI: https://doi.org/10.1021/c400093k

KUTZER, M. D. M.; DEVRIES, L. D.. Testbed for Multilayer Conformal Additive Manufacturing. Technologies, v.5, n.2, 2017. DOI: https://doi.org/10.3390/technologies5020025

LAPLUME, A. O.; PETERSEN, B.; PEARCE, J. M.. Global value 
chains from a 3D printing perspective. Journal of International Business Studies, v.47, n.5, p.595-609, 2016. DOI: https://doi.org/10.1057/jibs.2015.47

LAURETO, J. J.; PEARCE, J. M.. Open Source Multi-Head 3D Printer for Polymer-Metal Composite Component Manufacturing. Technologies, v.5, n.2, p.36-58, 2017. DOI: https://dx.doi.org/10.3390/technologies5020036

LE BOURHIS, F.; KERBRAT, O.; HASCOET, J. Y.; MOGNOL, P.. Sustainable manufacturing: evaluation and modeling of environmental impacts in additive manufacturing. The International Journal of Advanced Manufacturing Technology, v.69, n.9-12, p.1927-1939, 2013. DOI: https://doi.org/10.1007/s00170-013-5151-2

LE DUIGOU, A.; CASTRO, M.; BEVAN, R.; MARTIN, N.. 3D printing of wood fibre biocomposites: From mechanical to actuation functionality. Materials \& Design, v.96, p.106-114, 2016. DOI: https://doi.org/10.1016/j.matdes.2016.02.018

LIU, F. H.. Synthesis of biomedical composite scaffolds by laser sintering: Mechanical properties and in vitro bioactivity evaluation. Applied Surface Science, v.297, p.1-8, 2014. DOI: https://doi.org/10.1016/j.apsusc.2013.12.130

MASLOWSKI, M.; MIEDZIANOWSKA, J.; STRZELEC, K.. Natural rubber biocomposites containing corn, barley and wheat straw. Polymer Testing, v.63, p.84-91, 2017. DOI: https://doi.org/10.1016/i.polymertesting.2017.08.003

MEIRA, C. R.; CARVALHO, J.; PURQUERIO, B. M.; FORTULAN, C.mA.. Development of gypsum powder and binder for 3D print rapid prototyping. Cerâmica, v.59, n.351, p.401-408, 2013. DOI: https://dx.doi.org/10.1590/S036669132013000300009

MELO NETO, F. P.; BRENNAND, J. M.. Empresas socialmente sustentáveis: o novo desafio da gestão moderna. Rio de Janeiro: Qualitymark, 2004.

MENG, F.; ZHOU, Y.; LIU, J. Y.; WU, J.; WANG, G.; LI, R.; ZHANG, Y.. Thermal decomposition behaviors and kinetics of carrageenan-poly vinyl alcohol bio-composite film. Carbohydrate Polymers, v.201, p.96-104, 2018. DOI: https://doi.org/10.1016/i.carbpol.2018.07.095

MILEO, P. C.. Aplicações da celulose de palha de cana-deaçúcar: obtenção de derivados partindo de celulose branqueada e de biocompósitos com poliuretana obtida a partir de óleo de mamona (Ricinus communis L.). Dissertação (Mestrado em Conversão de Biomassa) - Universidade de São Paulo, Lorena, 2011. DOI:

https://doi.org/10.11606/D.97.2011.tde-24102012-120717

MOHANTY, A. K.; MISRA, M.; HINRICHSEN, G.. Biofibres, biodegradable polymers and biocomposites: An overview. Macromol. Mater. Eng., v.276-277, n.1, p.1-24, 2000. DOI: https://doi.org/10.1002/(SICI)1439-

2054(20000301)276:1<1::AID-MAME1>3.0.CO;2-W

MOHANTY, S.; NAYAK, S. K.. Dynamic and steady state viscoelastic behavior and morphology of MAPP treated $\mathrm{PP} / \mathrm{sisal}$ composites. Materials Science and Engineering, v.443, n.1-2, p.202-208, 2007. DOI: https://doi.org/10.1016/j.msea.2006.08.053
MURPHY, C. A.; COLLINS, M. N.. Microcrystalline cellulose reinforced polylactic acid biocomposite filaments for $3 \mathrm{D}$ printing. Polymer Composites, v.39, n.4, p.1311-1320, 2018. DOI: https://doi.org/10.1002/pc.24069

NAGARAJAN, V.; MOHANTY, A. K.; MISRA, M.. Perspective on Polylactic Acid (PLA) based Sustainable Materials for Durable Applications: Focus on Toughness and Heat Resistance. ACS Sustainable Chemistry \& Engineering, v.4, n.6, p.2899-2916, 2016. DOI:

https://doi.org/10.1021/acssuschemeng.6b00321

NAVARRETE, J. I. M.; HIDALGO-SALAZAR, M. A.; NUNEZ, E. E.; ARCINIEGAS, A. J. R.. Thermal and mechanical behavior of biocomposites using additive manufacturing. International Journal on Interactive Design and Manufacturing, v.12, n.2, p.449-458, 2018. DOI: https://dx.doi.org/10.1007/s12008$\underline{017-0411-2}$

NGO, T. T.; BLAIR, S.; KUWAHARA, K.; CHRISTENSEN, D.; BARRERA, I; DOMINGO, M.; SINGAMNENI, S.. Drug impregnation for laser sintered poly (methyl methacrylate) biocomposites using supercritical carbon dioxide. Journal of Supercritical Fluids, v.136, p.29-36, 2018. DOI: https://doi.org/10.1016/j.supflu.2018.01.030

NING, F.; CONG, W.; QIU, J.; WEI, J.; WANG, S.. Additive manufacturing of carbon fiber reinforced thermoplastic composites using fused deposition modeling. Composites Part B: Engineering, v.80, p.369-378, 2015. DOI: https://doi.org/10.1016/j.compositesb.2015.06.013

PETROVIC, V.; GONZALES, J. V. H.; FERRADO, O. J.; GORDILLO, J. D.; PUCHADES, J. R. B.; GINAN, L. P.. Additive layered manufacturing: sectors of industrial application shown through case studies. Int. J. Prod. Res., v.49, n.4, p.1071-1079, 2011. DOI: http://dx.doi.org/10.1080/00207540903479786

RAMIRES, E. C.. Biocompósitos a partir de matrizes poliméricas baseadas em lignina, tanino e glioxal reforçadas com fibas naturais. Tese (Doutorado em Ciências Físico-Químicas) - Universidade de São Paulo, São Paulo, 2010.

RAYNA, T.; STRIUKOVA, L.. From rapid prototyping to home fabrication: How 3D printing is changing business model innovation. Technological Forecasting and Social Change, v.102, p.214-224, 2016. DOI: https://doi.org/10.1016/i.techfore.2015.07.023

RÉQUILÉ, S.; LE DUIGOU, A.; BOURMAUD, A.; BALEY, C.. Peeling experiments for hemp retting characterization targeting biocomposites. Industrial Crops and Products, v.123, p.573-580, 2018.

https://doi.org/10.1016/j.indcrop.2018.07.012

RODRIGUES, V. P.; ZANCUL, E. S.; MANÇANARES, C. G.; GIORDANO, C. M.; SALERNO, M. S.. Manufatura aditiva: estado da arte e framework de aplicações. GEPROS. Gestão da Produção, Operações e Sistemas, v.12, n.3, p.1-34, 2017. DOI: https://doi.org/10.15675/gepros.v12i3.1657

SAYYAR, S.; OFFICER, D. L.; WALLACE, G. G.. Fabrication of 3D structures from graphene-based biocomposites. Journal of Materials Chemistry B, v.5, n.19, p.3462-3482, 2017. DOI: https://doi.org/10.1039/C6TB02442D 
SCAFFARO, B.; LOPRESTI, F.; BOTTA, L.. PLA based biocomposites reinforced with Posidonia oceanica leaves. Composites Part B: Engineering, v.139, p.1-11, 2018. DOI: https://doi.org/10.1016/j.compositesb.2017.11.048

SHIBATA, S.; CAO, Y.; FUKUMOTO, I.. Flexural modulus of the unidirectional and random composites made from biodegradable resin and bamboo and kenaf fibres. Composites Part A: Applied Science and Manufacturing. v.39, n.4, p.640-646, 2008. DOI: https://doi.org/10.1016/j.compositesa.2007.10.021

SINGH, J. I. P.; DHAWAN, V.; SINGH, S.; JANGID, K.. Study of Effect of Surface Treatment on Mechanical Properties of Natural Fiber Reinforced Composites. Materials Today: Proceedings, v.4, n.2, p.2793-2799, 2017. DOI: https://doi.org/10.1016/i.matpr.2017.02.158

SOUZA, S. F.; FERREIRA, M.; SAIN, M.; FERREIRA, M. Z.; PUPO, H. F.; CHERIAN, B. M.; LEÃO, A. L.. The use of curaua fibers as reinforcements in composites. Biofiber Reinforcements in Composite Materials, p.700-720, 2015. DOI: https://doi.org/10.1533/9781782421276.5.700

TARRÉS, Q.; MELB $\varnothing$, J. K.; DELGADO-AGUILAR, M.; ESPINACH, F. X.; MUTJÉ, P.; CHINGA-CARRASCO, G.. Biopolyethylene reinforced with thermomechanical pulp fibers: Mechanical and micromechanical characterization and its application in 3D-printing by fused deposition modelling. Composites Part B: Engineering, v.153, p.70-77, 2018. DOI: https://doi.org/10.1016/j.compositesb.2018.07.009

TRAN, D. T.; LEE, H. R.; JUNG, S.; PARK, M. S.; YANG, J. W.. Lipid-extracted algal biomass based biocomposites fabrication with poly (vinyl alcohol). Algal Research, v.31, p.525-533, 2018. DOI: https://doi.org/10.1016/i.algal.2016.08.016

TRINDADE, W. G.; HOAREAU, W.; RAZERA, I. A. T.; RUGGIERO, R.; FROLLINI, E.; CASTELLAN, A.. Phenolic Thermoset Matrix Reinforced with Sugar Cane Bagasse Fibers: Attempt to Develop a New Fiber Surface Chemical Modification Involving Formation of Quinones Followed by Reaction with Furfuryl Alcohol. Macromol. Mater. Eng., v.289, n.8, p.728-736, 2004. DOI: https://doi.org/10.1002/mame.200300320

VAEZI, M.; BLACK, C.; GIBBS, D. M. R.; OREFFO, R. O. C.; BRADY, M.; MOSHREFI-TORBATI, M.; YANG, S..

Characterization of New PEEK/HA composites with 3D HA network fabricated by extrusion freeforming. Molecules, v.21, n.6, p.1-22, 2016. DOI:

https://doi.org/10.3390/molecules 21060687

VALENCIA, G. A.; LUCIANO, C. G.; LOURENÇO, R. V.; SOBRAL. P. J. A.. Microstructure and physical properties of nanobiocomposite films based on cassava starch and laponite. International Journal of Biological Macromolecules, v.107, p.1576-1583, 2018. DOI: https://doi.org/10.1016/j.ijbiomac.2017.10.031
VENA, P. F.. Thermomechanical pulping (TMP), chemithermomechanical pulping (CTMP) and biothermomechanical pulping (BTMP) of bugweed (Solanum mauritianum) and Pinus patula. Thesis (Doctorate) - University of Stellenbosch, Stellenbosch, 2005.

VIEIRA, E. S.; GOMES, J. A. N. S.. A comparison of Scopus and web of science for a typical university. Scientometrics, v.81, n.2, p.587-600, 2009. DOI: https://doi.org/10.1007/s11192009-2178-0

VILELA, C.; SOUSA, A. F.; FREIRE, C. S. R.; SILVESTRE, A. J. D.; PASCOAL NETO, C.. Novel sustainable composites prepared from cork residues and biopolymers. Biomass and Bioenergy, v.55, p.148-155, 2013. DOI: https://doi.org/10.1016/j.biombioe.2013.01.029

WINNEBECK, K. H.. An abbreviated alternatives assessment process for product designers: a children's furniture manufacturing case study. Journal of Cleaner Production, v.19, n.5, p.464-476, 2011. DOI: https://doi.org/10.1016/j.jclepro.2010.10.008

WITTBRODT, B.; LAURETO, J.; TYMRAK, B.; PEARCE, J. M.. Distributed manufacturing with 3-D printing: a case study of recreational vehicle solar photovoltaic mounting systems. Journal of Frugal Innovation, v.1, n.1, 2015. DOI: https://doi.org/10.1186/s40669-014-0001-z

WONG, K. V.; HERNANDEZ, A.. A review of additive manufacturing. International Scholarly Research Network, ISRN Mechanical Engineering, p.1-10, 2012. DOI: http://dx.doi.org/10.5402/2012/208760

XU, W.; PRANOVICH, A.; UPPSTU, P.; WANG, X.; KRONLUND D.; HEMMING, J.; ÖBLOM, H.; MORITZ, N.; PREIS, M.; SANDLER, N.; WILLFÖR, S.; XU, C.. Novel biorenewable composite of wood polysaccharide and polylactic acid for three dimensional printing. Carbohydrate Polymers, v.187, p.51-58, 2018. DOI:

https://doi.org/10.1016/i.carbpol.2018.01.069

YAO, F.; WU, Q.; LEI, Y.; XU, Y.. Rice straw fiber-reinforced high-density polyethylene composite: Effect of fiber type and loading. Industrial Crops and Products, v.28, n.1, p.6372, 2008. DOI:

https://doi.org/10.1016/j.indcrop.2008.01.007

YOON, H. S.; LEE, J. Y.; KIM, H. S.; KIM, M. S.; KIM, E. S.; SHIN, Y. J.; CHU, W. S.; AHN, S. H.. A comparison of energy consumption in bulk forming, subtractive, and additive processes: review and case study. Int. J. of Precis. Eng. and Manuf.-Green Tech., v.1, n.3, p.261-279, 2014. DOI: https://doi.org/10.1007/s40684-014-0033-0

ZHANG, F.; WEI, M.; VISWANATHAN, V. V.; SWART, B.; SHAO, Y.; WU, G.; ZHOU, C.. 3D printing technologies for electrochemical energy storage. Nano Energy, v.40, p.418431, 2017. DOI: https://doi.org/10.1016/j.nanoen.2017.08.037

A CBPC - Companhia Brasileira de Produção Científica (CNPJ: 11.221.422/0001-03) detém os direitos materiais desta publicação. Os direitos referem-se à publicação do trabalho em qualquer parte do mundo, incluindo os direitos às renovações, expansões e disseminações da contribuiç̃o, bem como outros direitos subsidiários. Todos os trabalhos publicados eletronicamente poderão preservam os direitos autorais, mas não têm permissão para a publicação da contribuição em outro meio, impresso ou digital, em português ou em tradução. 